\title{
Effects of booms of sounding rockets in flowing plasmas
}

Cite as: Phys. Plasmas 26, 032902 (2019); https://doi.org/10.1063/1.5051414

Submitted: 08 August 2018. Accepted: 26 December 2018. Published Online: 11 March 2019

J. J. P. Paulsson (D), Y. Miyake (D), W. J. Miloch (D), and H. Usui (i)
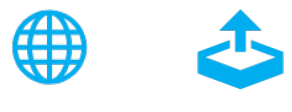

\section{ARTICLES YOU MAY BE INTERESTED IN}

Dynamics of ambipolarity

Physics of Plasmas 26, 032504 (2019); https://doi.org/10.1063/1.5091685

Numerical simulations of a dust grain in a flowing magnetized plasma

Physics of Plasmas 26, 043701 (2019); https://doi.org/10.1063/1.5089631

On the generalized formulation of Debye shielding in plasmas

Physics of Plasmas 26, 050701 (2019); https://doi.org/10.1063/1.5091949

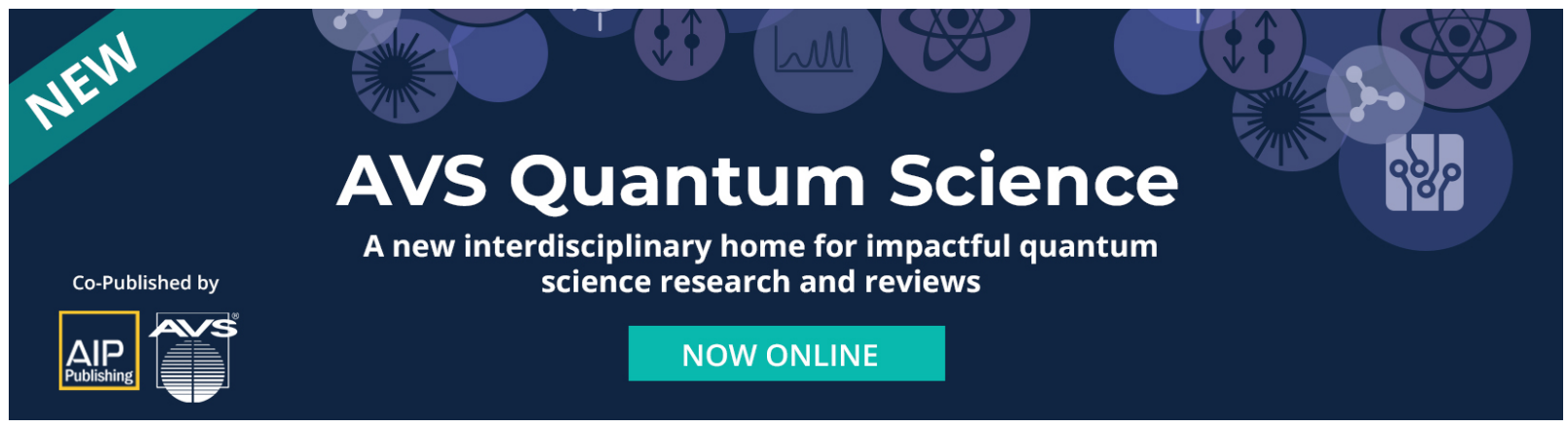




\title{
Effects of booms of sounding rockets in flowing plasmas
}

\author{
Cite as: Phys. Plasmas 26, 032902 (2019); doi: 10.1063/1.5051414 \\ Submitted: 08 August 2018 - Accepted: 26 December 2018 - Published Online: \\ 11 March 2019
}

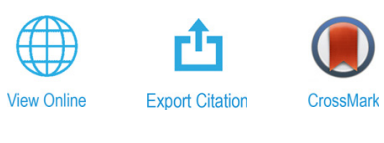

\section{J. J. P. Paulsson, ${ }^{1, a)}$ (D) Y. Miyake, ${ }^{2}$ (D) W. J. Miloch, ${ }^{1}$ (D) and H. Usui ${ }^{2}$ (D)}

\author{
AFFILIATIONS \\ ${ }^{1}$ Department of Physics, University of Oslo, Postboks 1048 Blindern, 0316 Oslo, Norway \\ ${ }^{2}$ Graduate School of System Informatics, Kobe University, 1-1 Rokkodai-cho, Nada-ku, Kobe 657-8501, Japan

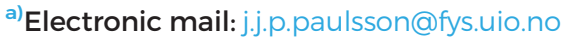

\begin{abstract}
Effects of booms of a sounding rocket on rocket charging and on the local plasma conditions are studied with numerical and analytical models accounting for flowing, ionospheric plasmas. Simulations are carried out with a first principles self-consistent Particle-In-Cell numerical code. It is shown that the booms can affect the charging of the payload and disturb the local plasma to a high degree. The boom-to-plasma potential, which varies with the position due to the convective electric field, can lead to significantly different currents, in particular electron currents, to the booms on either side of the rocket. The analytical models for the collected currents considered in this study show good agreement with the simulations. The potential difference between the plasma and the rocket can electrostatically focus the ions to create regions of enhanced ion density downstream of the rocket and can lead to an asymmetric wake. It is shown that ion focusing can be asymmetric between the booms due to the potential gradient. These effects can have implications for instrument placements and data analysis.
\end{abstract}

Published under license by AIP Publishing. https://doi.org/10.1063/1.5051414

\section{INTRODUCTION}

Charging of spacecraft in space is an important issue not only for protection of the payload circuits but also for performing in situ measurements with instruments onboard spacecraft. $^{1,2}$ Examples of such instruments are Langmuir probes, which for their operation often need to attain a specific electric bias voltage with respect to plasma. ${ }^{3,4}$ Since the payload provides the potential bias, it will be seen as ground by the probe. If the payload and spacecraft are electrically conducting, they will attain the same floating potential. ${ }^{2}$ Thus, for proper data analysis and operation of payload instruments, it is essential to have good knowledge on the spacecraft potential for plasma conditions relevant for particular space missions.

There are different space plasma environments, ranging from the ionosphere (relevant for sounding rockets), ${ }^{5-8}$ where the Debye length is generally shorter than the typical length scale of the payload, to the lobes of the geomagnetic tail or interplanetary space, ${ }^{9,10}$ where the Debye length can be much larger than the spacecraft. Using sounding rockets to study ionospheric plasmas by in situ measurements poses some unique problems, but it also benefits from the ability of launching a rocket in a relatively short time-span to investigate short-lived or fast traveling phenomena. ${ }^{5,8}$ In order to be able to correctly analyze data collected by the sounding rocket payload instruments, one needs to understand the charging of the payload and how the payload interacts with the surrounding plasma.

A charged ionospheric rocket will affect the plasma in its vicinity mainly through the electrostatic sheath or wake development. 111,12 The extent of the electrostatic sheath is usually of an order of the Debye length, while the extent of wake, which forms downstream of a rocket moving with respect to plasma, depends on the plasma flow and thermal speed. ${ }^{13-16}$ In an effort to avoid these effects, booms might be attached to the payload. Mounting instruments on such booms will improve the measurements by reducing the effects of plasma disturbed by the rocket body. This is certainly true regarding the electrostatic sheath, where already a short boom can place an instrument outside the rocket sheath. The wake, on the other hand, may extend very far downstream. In fact, Al'pert et al. ${ }^{11}$ indicated that the wake may be divided into two regions: (i) a region close to the body, which is dominated by the potential of the rocket body, and (ii) a region far away from the rocket, which is dominated by shadowing of ion trajectories in the phase-space. Depending on the thermal velocity of ions, the flow speed of 
plasma, and the rocket geometry, the extent of the second region may be of the order of hundreds of Debye lengths. ${ }^{17}$ Hence, attaching stable booms that are able to extend far enough from the rocket, so that instruments can be allowed to experience mostly undisturbed plasma, is a non-trivial task. Thus, a good knowledge of the rocket-plasma interaction is required to interpret data from such instruments.

Furthermore, due to the complex geometry of a rocket with the boom system attached, its complete interaction with the surrounding plasma is unpredictable by analytical approaches. This is valid not only for the plasma in its vicinity but also for charging of the payload. The main body of the payload is often much larger than the Debye length and shaped like a cylinder, whereas the thickness of the booms can often be comparable with or smaller than the Debye length. This means that the plasma currents collected by the main body and booms may need to be addressed by different approaches.

The convective electric field in the ionosphere, as experienced by the rocket, i.e., in the reference frame fixed to the rocket body, will lead to a potential difference between the tips of the booms if they are aligned with the vector components of the electric field. As a result, the potential difference between the plasma and the floating potential of the boom on either side of the rocket will be different and can be related to the electric field strength and the boom length. Not only will this affect the charging of the rocket, but it can also lead to asymmetries in plasma density distributions on different sides of the spacecraft.

In this work, we address the problem of the interaction of the ionospheric plasma with a cylindrically shaped rocket with booms, by employing self-consistent numerical simulations. The simulations are carried out with the Particle-In-Cell (PIC) computer code. We consider different plasma flow velocities and compare numerical results to analytical theories. We demonstrate that the presence of booms has non-trivial effects on the charging of the rocket and the plasma distribution in the vicinity of the rocket body.

\section{METHOD}

To study the role of booms in the rocket-charging and rocket-plasma interaction, we employ a PIC numerical code, EMSES. ${ }^{9}$ The details on the EMSES code are given in previous works. ${ }^{9}$ In particular, for a more comprehensive description of a similar setup as in the present study, see the study by Darian et $a{ }^{16}$ In the following, we provide only the main idea behind the algorithm and parameters used in this study.

In the PIC method, the plasma particles (i.e., electrons and ions) interact with each other via a computational grid that is used to calculate force fields. This reduces the computational cost and makes it feasible to simulate large plasma systems. ${ }^{18}$ The main computational cycle consists of (1) weighing numerical particles up to the nearest grid points, (2) solving the field equations and finding forces on the grid, (3) projecting forces from grid points onto particles, and (4) advancing particle trajectories according to the equations of motion. Thus, in the PIC method, we can study a self-consistent evolution of a system and base this study on first principles.
A rocket with booms is placed on a Cartesian and forms internal boundary conditions for the plasma particle trajectories (see Fig. 1). It is placed inside a simulation box of $39 \lambda_{\mathrm{D}}$ in each direction, where $\lambda_{\mathrm{D}}=\sqrt{\epsilon_{0} k_{\mathrm{B}} \mathrm{T}_{e} / n e^{2}}$ is the electron Debye length with $\epsilon_{0}, k_{\mathrm{B}}, \mathrm{T}_{e}, n_{0}$, and $e$ being the electron permittivity of vacuum, the Boltzmann constant, the electron temperature, the plasma density, and the electron charge, respectively. The sizes of the rocket and the booms are $14.8 \lambda_{\mathrm{D}}$ and $35.2 \lambda_{\mathrm{D}}$, respectively, ensuring that the main rocket body is introduced far from the boundaries and that there are no boundary effects affecting the boom charging. With respect to the field solver, we use the Dirichlet boundary conditions, and for our selected plasma parameters and considered plasma flow velocities, our simulations are stable, and we do not see any noticeable boundary effects on the wake formation.

Charged plasma particles hitting the surface of the rocket contribute to its surface charge. For computational reasons, it is not possible to simulate the actual size of a typical ionospheric sounding rocket with the booms, which often have a tip-to-tip length more than 1 meter. However, we use a scaled system which can be used as a model case, where the ratios between the Debye length and the rocket, as well as boom sizes, are maintained. The rocket surface is equipotential, which is achieved in the numerical simulation with the capacitance matrix method. ${ }^{9,18}$ The simulation domain is open for plasma particles and can account for the plasma flow. While EMSES is an electromagnetic code, in the present study, we work in the electrostatic approximation with an external static magnetic field.

The simulated plasma parameters are relevant for ionospheric plasma conditions and are summarized in Table I. The background plasma density is $n_{0}=2 \times 10^{11} \mathrm{~m}^{-3}$, the electron and ion temperatures are $T_{e}=3000 \mathrm{~K}$ and $\mathrm{T}_{i}=2000 \mathrm{~K}$, respectively, and the ion to electron mass ratio is $m_{i} / m_{e}=500$, which is large enough to separate the dynamics of electrons and ions but still allows for a reasonable time of simulations. The background magnetic field is $\mathrm{B}=50 \mu \mathrm{T}$. We consider the plasma flow velocities $v_{d}$ in the range of $M \in(0.5,8)$ with increments of 0.5 for each simulation, where $M=v_{d} / C_{s}$ is the Mach number and $C_{s}$ $=\sqrt{k_{\mathrm{B}} \mathrm{T}_{e} / m_{i}}$ is the sound speed. The flow is perpendicular to the rocket axis and booms, and the geometry and dimensions of the rocket are shown in Fig. 1, together with the coordinate system.

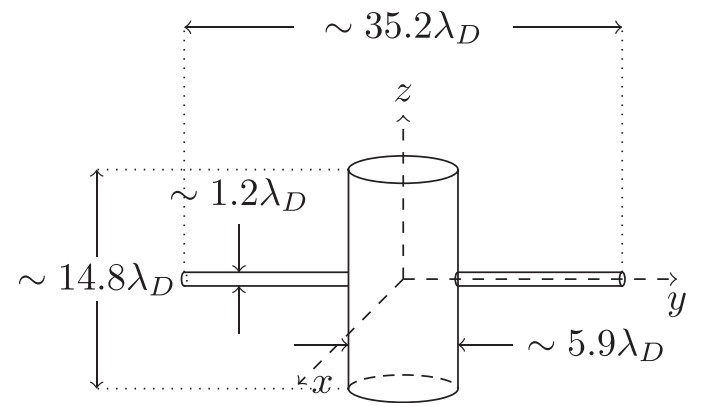

FIG. 1. Schematic of the simulated rocket and booms with their sizes and a coordinate system. 
TABLE I. List of parameters used in the simulation.

\begin{tabular}{lcc}
\hline \hline Parameter & Symbol & Value \\
\hline Plasma density $\left(\mathrm{m}^{-3}\right)$ & $n_{0}$ & $2 \times 10^{11}$ \\
Electron temperature $(\mathrm{K})$ & $T_{e}$ & 3000 \\
lon temperature $(\mathrm{K})$ & $T_{i}$ & 2000 \\
Mach number & $M$ & $0.5-8.0$ \\
Magnetic field $(\mu \mathrm{T})$ & $B$ & 50 \\
lon-to-electron mass ratio & $m_{i} / m_{e}$ & 500 \\
Debye length $(\mathrm{cm})$ & $\lambda_{D}$ & 0.845 \\
Box size $(\mathrm{cm})$ & $L$ & 32.95 \\
\hline \hline
\end{tabular}

The magnetic field vector is always pointing in the positive $z$ direction, and the plasma flow velocity $\mathbf{v}_{\mathbf{d}}$ is oriented in the positive $x$-direction, following the electric field, corresponding to the $\mathbf{E} \times \mathbf{B}$-drift in the positive $y$-direction. The main body of the rocket is aligned with the $z$-axis and the booms with the $y$-axis.

\section{RESULTS}

We perform a series of simulations to study the effects of booms on the rocket-plasma interaction according to parameters given in Table I. Figure 2 shows the simulation results of the ion density for four different Mach numbers. The plasma flow is in the positive $x$-direction. There are clear ion focusing effects at the end points of the booms, which can be attributed to the electrostatic bending of ion trajectories. ${ }^{19}$ In Fig. 2(a), there is a similar focusing behind Boom 1 and Boom 2 , but as the drift velocity increases, the focusing behind Boom 2 decreases, while it gets more pronounced behind Boom 1. This can be attributed to the convective electric field: With the increasing Mach number, while keeping the magnetic field constant, the electric field increases according to $\mathbf{E}=-\mathbf{v}_{\mathbf{d}} \times \mathbf{B}=v_{d} B \hat{\mathbf{y}}$, and hence, the potential drop between the plasma and the rocket gets larger for Boom 1 than for Boom 2. The potential difference between the booms and the plasma can be seen in Fig. 3, which shows a cut through the electrostatic potential along the booms and the center of the rocket in the $y$-direction.

The current collected by a conductive body depends on the difference between the electric potential of the object and the plasma. ${ }^{3,4}$ Therefore, along the booms, each unit section will collect a different current due to the potential gradient originating from the convective electric field. Assuming that the potential difference is $V_{b}-V_{p}<0$, where $V_{b}$ is the potential of the body and $V_{p}$ is the plasma potential, and that each circular segment constitutes a radial conservative electrical force field, for all parts of the boom, the electron current to a cylindrical part of the boom at position $y$ will be

$$
d \mathrm{I}_{e}=2 \pi r d y \mathrm{I}_{0 e} \exp \frac{e\left(\mathrm{~V}_{b}-\mathrm{V}_{p}(y)\right)}{k_{\mathrm{B}} \mathrm{T}_{e}},
$$

where $r$ is the radius of the boom, $d y$ is the length of the cylindrical section of the boom, $\mathrm{I}_{0 e}=e n_{e} \sqrt{k_{\mathrm{B}} \mathrm{T}_{e} / 2 \pi m_{e}}$ is the electron random thermal current, $V_{b}$ is the potential of the boom, and $\mathrm{V}_{p}(y)$ is the plasma potential at position $y$. Assuming that the convective electric field is the dominant factor determining the plasma potential, we can write

$$
\mathrm{V}_{p}(y)=-v_{d} B y
$$

Then, letting $d y$ go to zero and summing over all the cylindrical sections, i.e., sections from one boom edge $y_{0}$ to the other $y_{1}$, we obtain the total electron current to the boom as
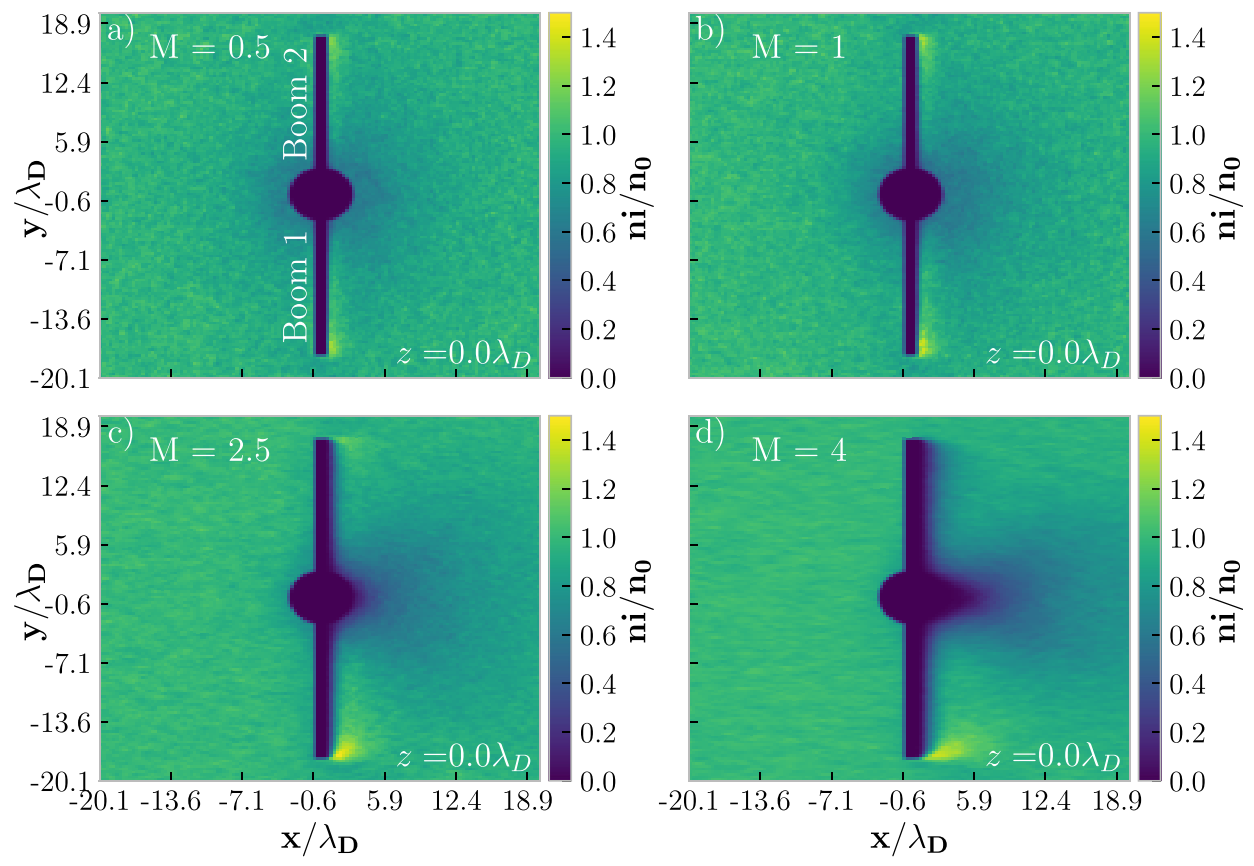

FIG. 2. Normalized distribution of the ion distribution in the $x$-y plane, at $z=0$ for different Mach numbers $M$ : (a) $M=0.5$, (b) $M=1.0$, (c) $M=2.0$, and (d) $M=4.0$. The plasma flow is in the positive $x$ direction. 


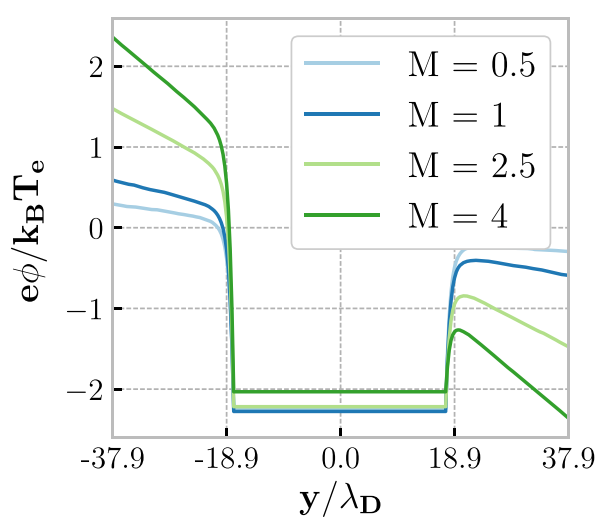

FIG. 3. Potential profile along $y$ for $z=x=0$. The potential difference between the body (the flat region around $y=0$ ) and the plasma potential is different for two ends of the booms.

$$
\begin{aligned}
\mathrm{I}_{e}= & 2 \pi r \mathrm{I}_{0 e} \exp \frac{e \mathrm{~V}_{b}}{k_{\mathrm{B}} \mathrm{T}_{e}} \int_{y_{0}}^{y_{1}} \exp \frac{e v_{d} \mathrm{By}}{k_{\mathrm{B}} \mathrm{T}_{e}} d y \\
= & 2 \pi r \mathrm{I}_{0 e} \frac{k_{\mathrm{B}} \mathrm{T}_{e}}{e v_{d} \mathrm{~B}} \exp \left(\frac{e \mathrm{~V}_{b}}{k_{\mathrm{B}} \mathrm{T}_{e}}\right)\left(\exp \left(\frac{e}{k_{\mathrm{B}} \mathrm{T}_{e}} v_{d} B y_{1}\right)\right. \\
& \left.-\exp \left(\frac{e}{k_{\mathrm{B}} \mathrm{T}_{e}} v_{d} \mathrm{~B} y_{0}\right)\right) .
\end{aligned}
$$

We can see that the electron current is a non-linear function of the ratio between the drift velocity and the electron temperature: $\mathrm{I}_{e} \sim \mathrm{T}_{e} / v_{d} \exp \left(v_{d} / \mathrm{T}_{e}\right)$. In the left panel of Fig. 4 , we have compared the electron currents to the boom as measured in the simulation with Eq. (3). The simulation and the analytical approximation show good agreement; however, there is a slight offset.

The collection of ions by finite-sized objects is often more involved. ${ }^{20}$ However, as the plasma flow velocity increases, one can expect that the cold ion approximation can provide an estimate of the ion current to a higher degree of accuracy. Assuming ions with no thermal motion, the flux to the booms can be expressed as

$$
I_{i}=2 r \operatorname{Len}_{i} v_{d}
$$

where $L$ is the boom length. We will refer to this current as the ram current.
Hoegy and Wharton ${ }^{21}$ derived an expression for an approximate ion current to a cylinder, where they also include ion thermal effects

$$
I_{i}=2 \pi r L_{0 i} \frac{2}{\sqrt{\pi}}\left(\left|V_{b}-V_{p}\right|+S_{d}^{2}+\frac{\left|V_{b}-V_{p}\right|+S_{d}^{2} / 2}{\left|V_{b}-V_{p}\right|+S_{d}^{2}}\right)^{1 / 2},
$$

where $\mathrm{I}_{0 i}=e n_{i} \sqrt{k_{\mathrm{B}} \mathrm{T}_{i} / 2 \pi m_{i}}$ and $\mathrm{S}_{d}=v_{d} / v_{\text {thi }}$, where $v_{\text {thi }}$ $=\sqrt{2 k_{\mathrm{B}} \mathrm{T}_{i} / m_{i}}$.

We compare these two approximations with the numerical results in Fig. 4. The right panel of Fig. 4 shows the ion current collected by the two booms, as well as the ion ram current of Eq. (4) and Hoegy and Wharton's ion current calculated using Eq. (5). For low $\mathrm{M}$, the ion current differs from the ram current approximation significantly, while Hoegy and Wharton's ion current is consistently closer to the numerical results. As $M$ increases, both analytical results get closer to the simulation results. Note that there is also, albeit small, an increasing difference in the ion currents to the two booms with increasing $M$.

The currents to the main body are shown in the left panel of Fig. 5 together with the ion currents given by Eqs. (4) and (5). One can see that both the ion and electron currents become closer to the analytical results with increasing $M$. Note that there is a slight imbalance between the ion and the electron currents to the main body, which increases with increasing $M$, and at $M=8$, the difference between the ion and the electron current is significant.

The floating potential of the body, i.e., the potential of the rocket body relative to the plasma, at which all the currents cancel out, is shown in the right panel of Fig. 5 with the plasma potential of zero as reference. Note from Fig. 3 that the plasma potential is different for each part of the boom; however, at the main body center, the plasma potential is zero. The potential starts to dip towards more negative when the flow transitions from subsonic to supersonic speeds, and at around $M=1.5$, it starts to increase. Note the increase in the body potential after the turning point is not linear, even though the ion current remains linear as can be seen in the right panel of Fig. 4.

As can be seen in Fig. 2 showing the ion density, the plasma around the rocket is disturbed. All parts of the rocket disturb the plasma, but the booms and the main body have different effects. Long and thin booms tend to focus ions, and this effect
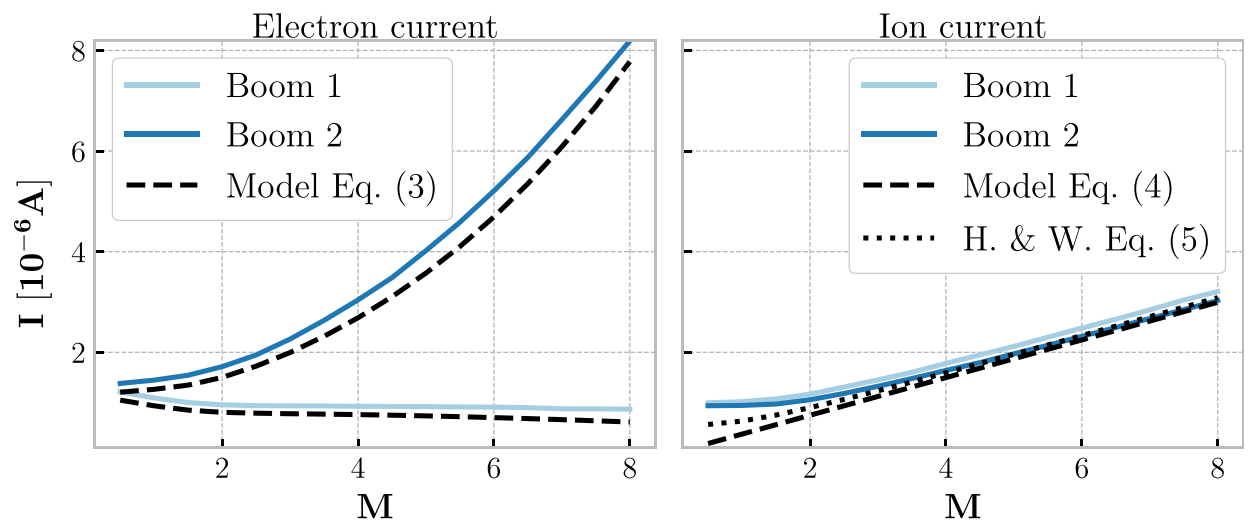

FIG. 4. Electron (left panel) and ion (right panel) currents to the booms versus the Mach number. Left panel: the electron currents from the simulations to the two booms (solid lines) and from the model given by Eq. (3) (dashed lines). Right panel: the ion currents from the simulations to the two booms (solid lines) and from the ion ram current model (dashed line) Eq. (4), as well as Hoegy and Wharton's model (dotted line) Eq. (5). 

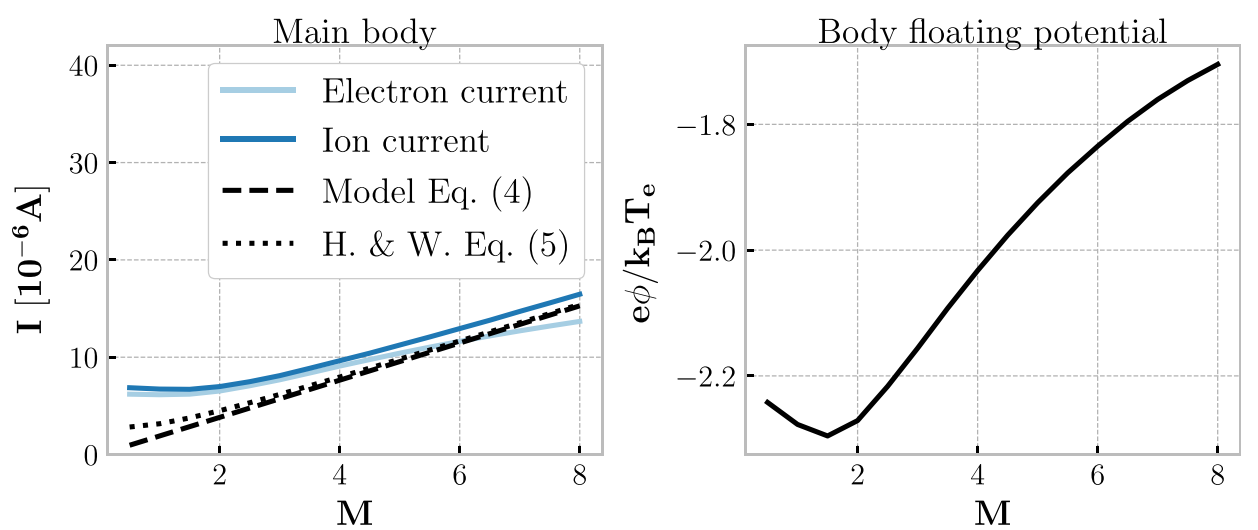

FIG. 5. Electron and ion currents to the main body (left panel) and the body floating potential (right panel) versus the Mach number. Left panel: the electron (solid light blue line) and ion (solid dark blue line) currents from the simulation and the ion ram current model (dashed line) Eq. (4), as well as Hoegy and Wharton's model (dotted line) Eq. (5). becomes more asymmetric with the increasing Mach number. In Fig. 2(a), we see a weak focusing downstream of the booms with small asymmetry, but in panel (d), there is a significant difference in the ion distribution behind the two booms. In Fig. 6, the ion density profiles across the booms at two different distances from the origin are shown. In Fig. 6(a), we see that the maximum ion focus is at $M=1$ at the tip of Boom 1, but as $M$ increases, the maximum in the ion density decreases and the enhanced ion density region extends to larger distances downstream of the boom. Also, note that no ion depletion is observed. In panel (c), which corresponds to the part of Boom 1 that is closer to the main body, the ion focusing is much less pronounced and is in fact not present at all for $M=4$. We can also see that a depletion in plasma density has occurred behind this part of the boom. For Boom 2, at the tip (panel b), significant focusing is present only for $M=1$, and almost no wake is formed for any case, except for $M=4$. In panel (d), for the part of Boom 2 that is closer to the main body, there is little enhancement in the ion density, but the ion depletions get more pronounced.

The corresponding profiles of ion density across the main body along the $x$-direction are shown in Fig. 7. The wakes extend further, up to several Debye lengths, downstream of the main body with increasing $M$. Upstream of the main body, the sheath extends farther for lower $M$.

Finally, the potential profile downstream of the booms at increasing distances from the surface of the main body is shown in Fig. 8. Note that the scale of the vertical axis changes in each of the panels. In panel (a), one can see the asymmetry in potential which can be attributed to the ion distribution behind the booms. For $M=4$, the potential reaches positive values on the
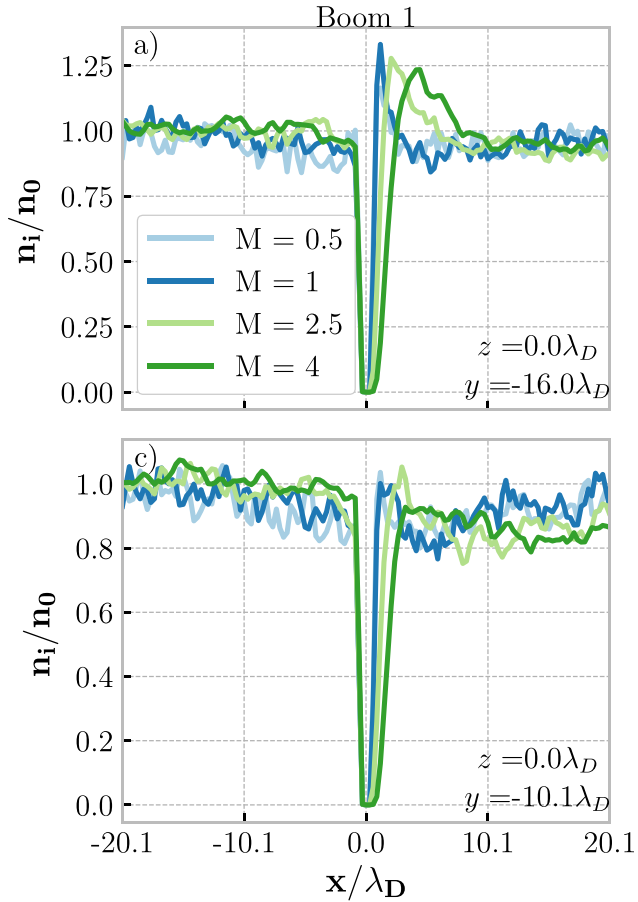
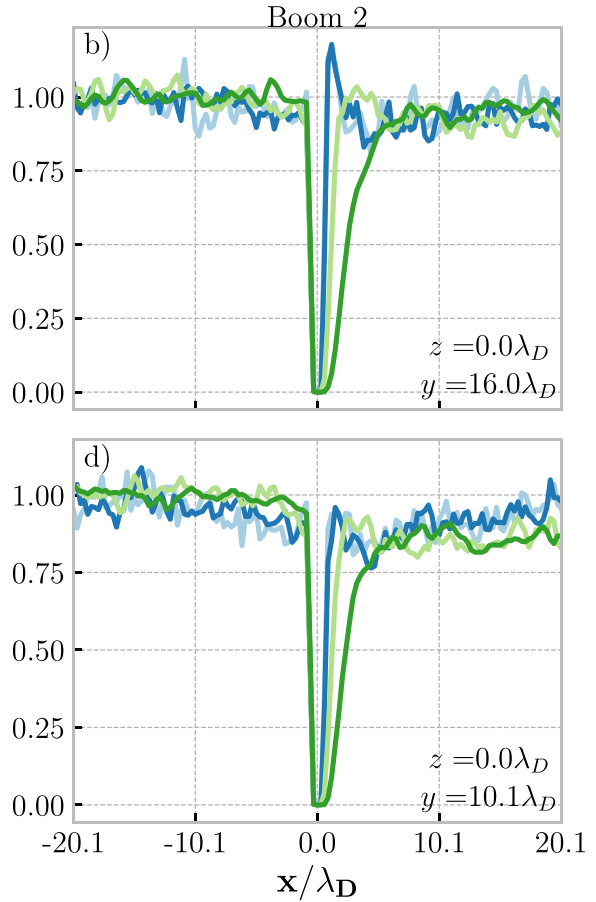

FIG. 6. Profiles of the normalized ion density $n_{i} / n_{0}$ along the $x$-axis for fixed $y$ and $z$ across the two booms at different distances from the origin and for different Mach numbers. See Fig. 2 for coordinates for the booms. 


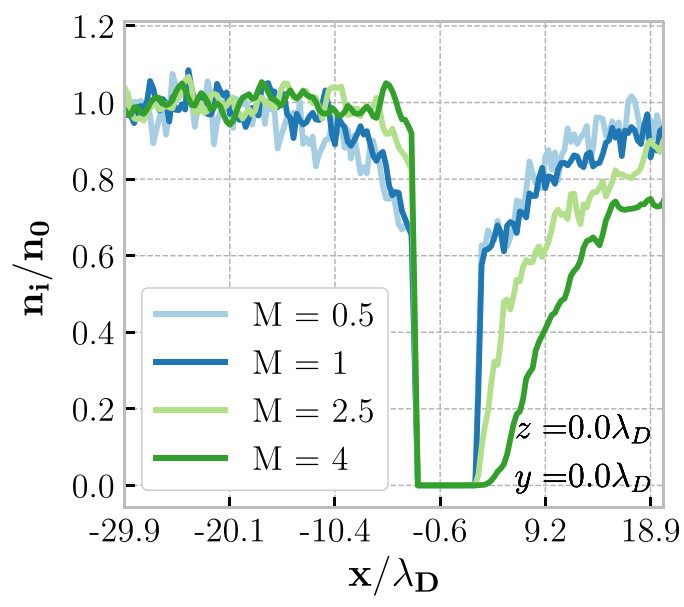

FIG. 7. Profiles of the normalized ion density $n_{i} / n_{0}$ along the $x$-axis at fixed $y=0$ and $z=0$ across the center of the main body for different Mach numbers. left-hand side of the wake at this distance. For $M=2.5$, there is still a small elevation of the potential, but it remains negative. The potential enhancement disappears at lower M. In panel (b), further downstream, such an enhancement in potential is not present for any cases except for $\mathrm{M}=4$ where a small bump is visible. For panel (c), the wake is relatively symmetric for all cases, with only slight asymmetries visible. At about 20 Debye lengths, in panel (d), we see that some asymmetries in potential depletion start to develop, especially for $M=4$. In panel (e), there is an asymmetry of the wake for $M=4$. Finally, for panel (f), the wake for $M=0.5,1$, and 2.5 has almost vanished, while for $M=4$, we can see a region in the wake where the potential has relative enhancement. However, at such large distances, the potential variations are rather small, as also reflected in the scale of the vertical axis.

\section{DISCUSSION}

A conductive body in the ionosphere will collect and emit currents (e.g., plasma, photoemission, and secondary emission currents) and will attain a potential relative to the plasma. This floating potential will act in such a way that all currents will balance and the net current to the surface vanishes. ${ }^{1,2}$ Sounding
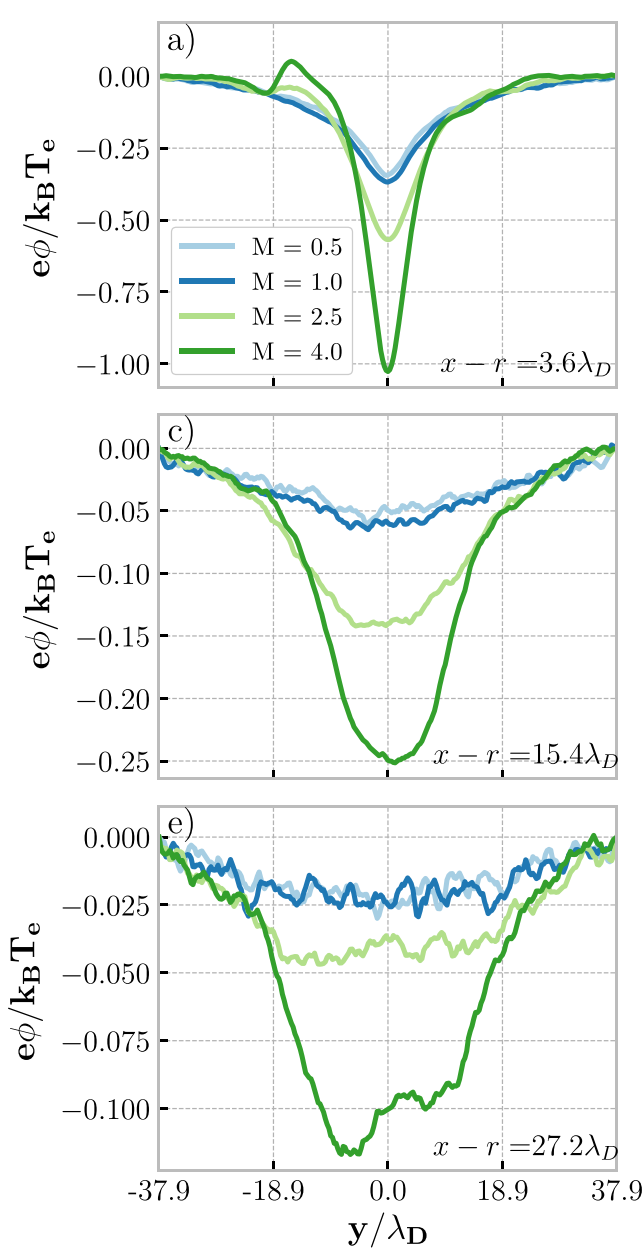
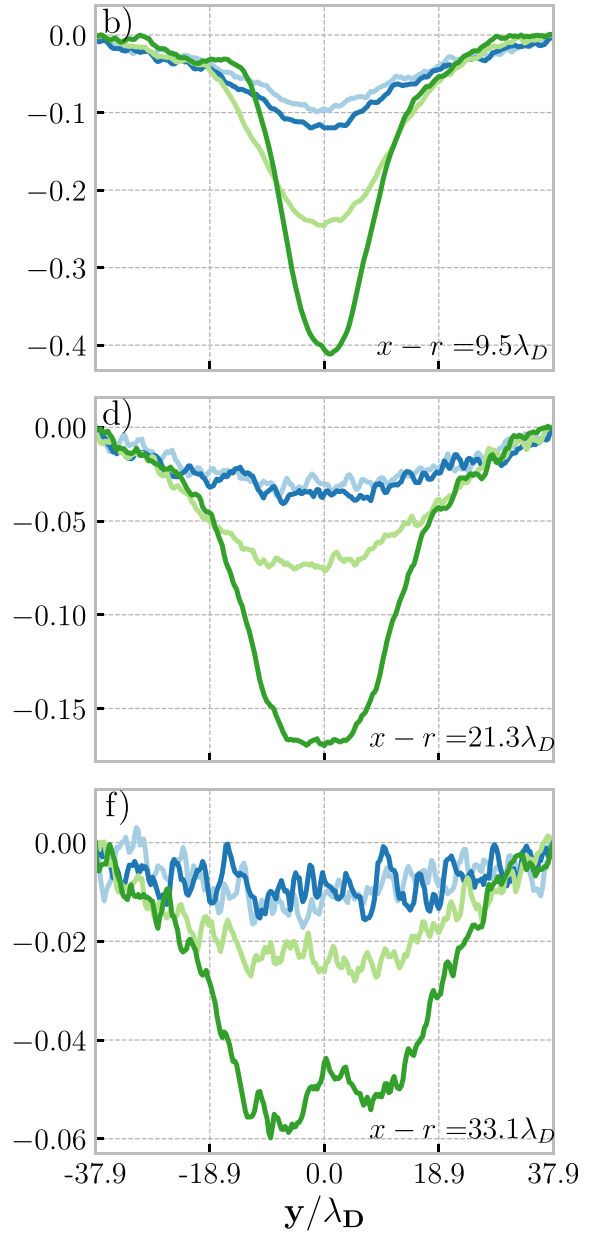

FIG. 8. The scaled potential profile behind the rocket taken along the $y$-axis, $z=0$ and at different distances from the rocket surface for different Mach numbers. Note that the scale of the vertical axis is different in each of the panels. 
rockets in the ionosphere usually attain negative floating potentials since the relative contribution of the photoemission and secondary electron emission currents is small, ${ }^{22}$ and thus, we have omitted them in our analysis. The main currents in the ionosphere driving the floating potential are then the ion and electron collection currents. In the polar ionosphere, there is often significant particle precipitation, which might affect the charging and the wake formation, but we do not consider it in the present study.

From Fig. 2, it is evident that the booms will have a significant effect on the ion density distribution around the rocket. In this figure, we have also restricted the analysis to the maximum of $M=4$ since higher flow velocities are unrealistic for sounding rockets. For low $M$, i.e., panels (a) and (b), the main effect around the booms is focusing of ions near each tip, where the electric field is the strongest, and as it can be inferred from Fig. 8, this has a negligible effect on the wake. In Figs. 6(a) and 6(b), the focusing is similar between the two cases but is slightly weaker around Boom 2. Ion focusing depends on the strength of the electric field in the vicinity of the surface, and as one can see in Fig. 3, the potential differences between the plasma and booms at the edges are similar for $M=0.5$ and 1 , implying similar ion acceleration for the two cases. However, in Figs. 2(c) and 2(d), ion focusing behind Boom 2 is smaller. Hence, ion focusing around the tips of the booms is the edge effect, where one can expect the strongest electric fields and ion acceleration, which is further enhanced by the potential difference due to the convective electric field.

When $M$ increases, asymmetry in ion focusing around Boom 1 and Boom 2 develops. Furthermore, these enhanced density regions for $M=2.5$ and $M=4$ have lower peaks and extend further than for smaller $M$, as shown in Fig. 6(a). This asymmetry and the difference in focusing are due to large electric fields, as is shown in Fig. 3, creating a significant difference in the potential differences at the edges of the two booms. Away from the edge of Boom 1 [panel (c) of Fig. 6], there are no significant focusing effects. As stated above, this is because focusing is mainly the edge effect due to the booms. However, for the focusing to occur, there should be a sufficient potential difference, as can be inferred from comparing the cases with low M. For Boom 2, instead of focusing, a geometric wake characterized by ion depletion due to absorption by the surface is formed (see Fig. 6 again). This geometric wake gets affected at higher $M$ by ion focusing at boom edges, which is a dynamic wake effect.

Since the booms are connected electrically to the main body, they will have an effect on the charging of the whole rocket. The ion and electron currents are different for each of the booms, as it can be seen in Fig. 4. The ion currents to the two booms are very similar for all considered flow velocities. At low $\mathrm{M}$, the currents are significantly different from the cold ion ram current given by Eq. (4). This can be attributed to the thermal motion of ions. Thermal ion motion is incorporated in Hoegy and Wharton's model, ${ }^{21}$ which indeed shows a better agreement with simulations. This is a similar result as from the study of McMahon et al. ${ }^{23}$ Small differences in the current to the booms might be attributed to different potential differences between the plasma and booms for each section of the booms due to the potential gradient, as well as edge effects.

The electron currents to the booms are very different between the booms as compared to the ion currents, and this difference increases with $M$ (see Fig. 4 again). Since electrons generally adjust to the potential much more efficiently than ions, the difference in electron currents to the two booms can again be explained by the potential gradient, leading to different potential differences between the booms and the plasma. The potential drop for Boom 2 is lower than for Boom 1 (see again Fig. 3), which is also reflected in the currents. Using Eq. (3), we derive an analytical expression of the total electron currents to the booms taking into account different potential drops between the plasma and the surface of the boom for each section of the boom. Our result from Eq. (3) is in good agreement with the simulation results but with a slight offset. This offset can be attributed to the fact that Eq. (3) is derived without considering the edge of the booms. In general, Eq. (3) can be used for any cylinder in a plasma that is aligned with the electric field when its potential is lower than the plasma potential, and the electrons are Boltzmann distributed.

For the main body, the electron and ion currents differ significantly from Eqs. (4) and (5) at low flow speeds. We attribute this to the currents to the top and the bottom of the cylinder, that is, Eqs. (4) and (5) are approximations using infinitely long cylinders with no edges as their geometry. For a cylinder with no booms, the electron and ion currents will cancel each other out so that the cylinder draws no net current from the plasma; however, we see that for high $M$, the electron and the ion current become significantly different. The ion current is still dominated by the ram current, but the electron current, which is much more sensitive to the potential of the object, reflects the very high increase in the electron currents which Boom 2 collects at high $M$. That is, the main body's electron current adjusts in order to maintain zero net current.

With subsonic plasma flow speeds, there will be a significant ion current contribution from all sides of the rocket, but as the flow speed increases, less and less ions will be collected on the surface of the rocket facing downstream due to the development of a wake. In other words, the collection cross-section changes with increasing $M$. As can be seen in the right panel of Fig. 5, the floating potential starts by becoming more negative. At around $M=1.5$, the potential starts to become less negative. This is due to the fact that the ion ram current becomes more significant as compared to the electron thermal current. The potential starts to increase at a lower rate with higher $M$ since Boom 2 collects an increased electron current.

It is evident that assumptions have been made regarding some of the plasma parameters in this study. For example, the ion mass is much lower than the oxygen, one of the main ion species of the ionosphere, which creates much stronger convective electric fields than those typically seen in the ionosphere. ${ }^{8,24}$ However, note that for collection and ion focusing, one of the important factors is the potential difference between the plasma and the surface of the collector. Since the rocket 
model in our simulation has very short booms, the potential difference is still in a realistic regime. ${ }^{25}$ One factor that is not represented well in this simulation is the force exerted on the ions by the electric potential of the body, which should in fact be much stronger than in reality. This might have an effect on the ion focusing and the asymmetry of the wake structures behind the rocket.

Understanding local plasma disturbances and charging of spacecrafts such as sounding rockets is important in order to estimate the validity of measurements done by instruments on-board. ${ }^{16,25}$ As shown in our study, measurements by rockets with instruments mounted on booms can experience difficulties due to local plasma disturbances. Thus, one needs to place instruments on booms in such a way as to minimize the risk of contaminating the data. Otherwise, the data may not correspond to undisturbed plasma conditions. Due to the complex nature of the object-plasma interactions, it is not trivial to provide a generic model for any kind of additional structural elements to a sounding rocket and their impact on the measurements. This study provides insights into how booms will affect rocket-plasma interactions. Not only is there a strong asymmetry in the current collection from the booms, which can affect circuitry and the overall floating potential, but there is also a possibility of ion-focusing. Ion focusing can extend far downstream and may impact the measurements. As shown by this study, instruments further from the tips of the booms might be less affected. The results can also be applied to explain artifacts in data from sounding rocket experiments or similar spacecrafts.

\section{CONCLUSION}

With Particle-in-Cell simulations and analytical models, we have studied a cylindrically shaped rocket with booms in flowing ionospheric plasmas. It has been revealed that booms can have a significant effect on both the charging of the payload and the disturbances of plasma in its vicinity. The electron and the ion currents to the booms are well approximated by analytical models, while for the main body, there are significant contributions from the top and the bottom of the rocket. Due to the potential gradient in flowing magnetized plasma, the booms collect different ion and electron currents, which are then compensated by the currents to the main body. The difference between the ion and the electron currents increases with increasing flow velocity.

Edge effects on the booms lead to ion focusing. For small Mach numbers, it is contained within a small distance behind the booms. With increasing flow velocity, the focusing is present at a significant distance downstream of the booms, which can be reflected in perturbations in the profile of the wake. Since most of the aspects of this study correspond, to a reasonable degree, to realistic sounding rocket experiment plasma conditions, the results can assist in a proper placement of instruments on such booms.

\section{ACKNOWLEDGMENTS}

This work is the result of the Japan-Norway Partnership on Space Science Simulations, funded by the Norwegian Center for International Cooperation in Education SiU, Grant Nos. UTF2014/10043 and UTF-2016-long-term/10054, and is a part of the 4DSpace Strategic Research Initiative at the University of Oslo. This study was supported in part by the Research Council of Norway under Grant No. 240000. The authors would also like to thank the participants of the Oslo-Kobe Workshop 2017 for their helpful contributions and advancements on subjects similar to the one presented in this study.

\section{REFERENCES}

${ }^{\mathbf{1} E . ~ C . ~ W h i p p l e, ~ R e p . ~ P r o g . ~ P h y s . ~ 44, ~} 1197$ (1981).

${ }^{2}$ H. B. Garrett, Rev. Geophys. 19, 577, https://doi.org/10.1029/ RG019i004p00577 (1981).

${ }^{3}$ H. M. Mott-Smith and I. Langmuir, Phys. Rev. 28, 727 (1926).

${ }^{4}$ L. Schott, Plasma diagnostics using electrical probes, in Reactions Under Plasma Conditions, edited by M. Venugopalan (John Wiley and Sons, 1971), Vol. 1, pp. 515-542.

${ }^{5}$ J. Moen, K. Oksavik, T. Abe, M. Lester, Y. Saito, T. A. Bekkeng, and K. S. Jacobsen, Geophys. Res. Lett. 39, L07104, https://doi.org/10.1029/ 2012GL051407 (2012).

${ }^{6}$ K. R. Svenes, J. Trøim, B. N. Maehlum, M. Friedrich, K. M. Torkar, G. Holmgren, and W. F. Denig, Planet. Space Sci. 38, 653 (1990).

${ }^{7}$ D. A. Lorentzen, J. Moen, K. Oksavik, F. Sigernes, Y. Saito, and M. G. Johnsen, J. Geophys. Res.: Space Phys. 115, A12323, https://doi.org/ 10.1029/2010JA015710 (2010).

${ }^{8}$ A. Spicher, A. A. Ilyasov, W. J. Miloch, A. A. Chernyshov, L. B. N. Clausen, J. I. Moen, T. Abe, and Y. Saito, J. Geophys. Res.: Space Phys. 121, 10466, https://doi.org/10.1002/2016JA022999 (2016).

${ }^{9}$ Y. Miyake and H. Usui, Phys. Plasmas 16, 062904 (2009).

${ }^{10}$ A. I. Eriksson, I. A. D. Engelhardt, M. André, R. Boström, N. J. T. Edberg, F. L. Johansson, E. Odelstad, E. Vigren, J.-E. Wahlund, P. Henri, J.-P. Lebreton, W. J. Miloch, J. J. P. Paulsson, C. S. Wedlund, L. Yang, T. Karlsson, R. Jarvinen, T. Broiles, K. Mandt, C. M. Carr, M. Galand, H. Nilsson, and C. Norberg, Astron. Astrophys. 605, A15 (2017).

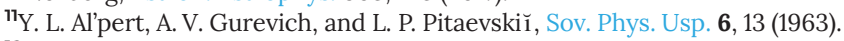

${ }^{12}$ J. Wang and D. E. Hastings, Phys. Fluids B: Plasma Phys. 4, 1615 (1992).

${ }^{13}$ A. V. Gurevich, L. V. Pariskaya, and L. P. Pitaevski, Sov. J. Exp. Theor. Phys. 36, 274 (1973); http://www.jetp.ac.ru/cgi-bin/e/index/e/36/2/p274?a=list.

${ }^{14}$ V. C. Liu, Prog. Aerosp. Sci. 16, 273 (1975).

${ }^{15}$ U. Samir, M. First, E. J. Maier, and B. Troy, J. Atmos. Terr. Phys. 37, 577 (1975).

${ }^{16}$ D. Darian, S. Marholm, J. J. P. Paulsson, Y. Miyake, H. Usui, M. Mortensen, and W. J. Miloch, J. Geophys. Res.: Space Phys. 122, 9603, https://doi.org/ 10.1002/2017JA024284 (2017).

${ }^{17}$ E. Engwall, A. I. Eriksson, and J. Forest, Phys. Plasmas 13, 062904 (2006).

${ }^{18}$ J. E. R. W. Hockney, Computer Simulation Using Particles (A. Hilger, 1988).

${ }^{19}$ W. J. Miloch and D. Block, Phys. Plasmas 19, 123703 (2012).

20 J. G. Laframboise and L. W. Parker, Phys. Fluids 16, 629 (1973).

${ }^{21}$ W. R. Hoegy and L. E. Wharton, J. Appl. Phys. 44, 5365 (1973).

${ }^{22}$ R. J. L. Grard, J. Geophys. Res. 78, 2885, https://doi.org/10.1029/ JA078i016p02885 (1973).

${ }^{23}$ J. C. McMahon, G. Z. Xu, and J. G. Laframboise, Phys. Plasmas 12, 062109 (2005).

${ }^{24}$ F. S. Mozer and P. Bruston, J. Geophys. Res. 72, 1109, https://doi.org/ 10.1029/JZ072i003p01109 (1967).

${ }^{25}$ J. J. P. Paulsson, A. Spicher, L. B. N. Clausen, J. I. Moen, and W. J. Miloch, J. Geophys. Res.: Space Phys. 123, 9711, https://doi.org/10.1029/ 2017JA025004 (2018). 